\title{
2. Arts in/as Education Practice
}

2.1. Embracing the maelstrom: Arts Therapy Training form the Edges of the Whirlpool by Kathrin Marks

2.2. Layers of reflexive learning by Jennifer Berlingieri

2.3. Earthbound by Bettina Evans

2.4. A making conversation by Stacey Bush and Amanda Woodford

2.5. Creative dialogue Jan Allen and Ariel Moy

Deb, it is the thread of arts in/as education practice which rises to the surface in this second group. In these works there is reflection on the work of balancing the roles and relationships between student and educator. An example of how the arts are used in teaching practice toward meeting learning outcomes. Two examples of teaching are demonstrated by creative engagement with the process and content of specific subjects. Finally, an example of how creative responding is used as a teaching tool in doctoral supervision.

When I read these, Stacey, I breathe in the passion and commitment of educators and students bringing themselves present again and again, being willing to be reflexive and reflective, to engage in emergence, to hold lightly, to engage multiple intelligences and modalities, to experiment and experience, to collaborate, and to invite their work to be evocatively tactile. 